\title{
Quadratic Gaussian Source Broadcast with Individual Bandwidth Mismatches
}

\author{
Louis Tan and Ashish Khisti \\ Dept. of Electrical \& Computer Engineering \\ University of Toronto \\ Toronto, ON M5S 3G4 Canada \\ \{ltan, akhisti\}@comm.utoronto.ca
}

\author{
Emina Soljanin \\ Bell Labs, Alcatel-Lucent \\ Murray Hill NJ 07974, USA \\ emina@alcatel-lucent.com
}

\begin{abstract}
We study the problem of broadcasting a Gaussian source over a Gaussian broadcast channel to two users with individual source-channel bandwidth mismatches, under a quadratic distortion measure. Specifically we study the tradeoff between the achievable distortion pairs between the two users as a function of the two bandwidth-expansion factors as well as the two signalto-noise ratios. The case when the bandwidth-expansion factors of the two users are identical has been well studied in the literature and to our best knowledge remains an open problem. Surprisingly, when the bandwidth expansion factors are different, we characterize a range of values where both the users simultaneously attain their point-topoint optimal distortion. Furthermore in the high signal-tonoise ratio regime, this set includes nearly all points where the weaker user has the higher bandwidth expansion factor. In other cases, we propose an achievable tradeoff between the distortion pairs.
\end{abstract}

\section{INTRODUCTION}

Consider the problem of sending a bandlimited Gaussian source over an additive bandlimited Gaussian noise channel. Suppose that we are unclear about the exact channel noise variance, however we still require that the encoder guarantees certain levels of reconstruction quality depending on the channel noise actually realized when the system is operational. For example, we design a single encoder and require that it achieves a low distortion for low noise power while relaxing our requirements and allowing for a higher distortion when experiencing higher noise power. This problem has been treated in the literature by considering a single transmitter that in fact broadcasts simultaneously to multiple receivers, each with different signal to noise ratios [1]-[3]. Given that the source and channel bandwidths are $W_{s}$ and $W_{c}$ $(\mathrm{Hz})$ respectively, we typically sample the source at a rate of $2 W_{s}$ samples per second, and subsequently encode the samples to be sent over the discrete-time Gaussian channels at a rate of $2 W_{c}$ channel uses per second. The associated bandwidth expansion factor is $b=W_{c} / W_{s}$ channel uses per source sample and for a given $b$ and the signal-to-noise ratios, a tradeoff between the achievable distortion pairs at the two users is of interest.

Now consider a variation on this problem. Imagine that we require a low distortion even when experiencing a weaker channel but we are willing to wait longer, i.e., observe more channel symbols before making a reconstruction. That is, we have the option of trading distortion for delay. In this setup, the transmitter continues broadcasting and each receiver continues listening until the distortion is sufficiently small. Such a problem can be modelled as an extension of the model considered in [1]-[3] where each receiver has a different bandwidth expansion factor.

In [4] a single server streaming model has been introduced. A server wishes to communicate a source sequence to a group of heterogeneous users over a broadcast channel. The underlying channels are modelled as erasure channels and each user's channel has a certain loss probability. Furthermore each user is only interested in retrieving a certain fraction of source packets. The transmitter continuously broadcasts coded packets and each receiver waits until it is able to retrieve sufficiently many packets of the underlying source. While the focus in [4] is to optimize the degree distribution of a rateless codes for this application, it can also be formulated as a joint source-channel coding problem for sending a binary source over a binary erasure broadcast channel with an erasure distortion measure [5]. In the present paper we only focus on the Gaussian version of the problem.

\section{Problem Formulation}

The problem is illustrated in Fig. 1. We consider a memoryless stationary Gaussian source $\{S(i)\}_{i=1,2, \ldots}$ producing symbols according to $\mathcal{N}\left(0, \sigma^{2}\right)$, which we wish to communicate to two users. Denote the vector $(S(1), S(2), \ldots, S(k))$ as $S^{k}$. The source is communicated by a block encoding function that maps the length $k$ source sequence $S^{k}$, to a length $n$ channel input 


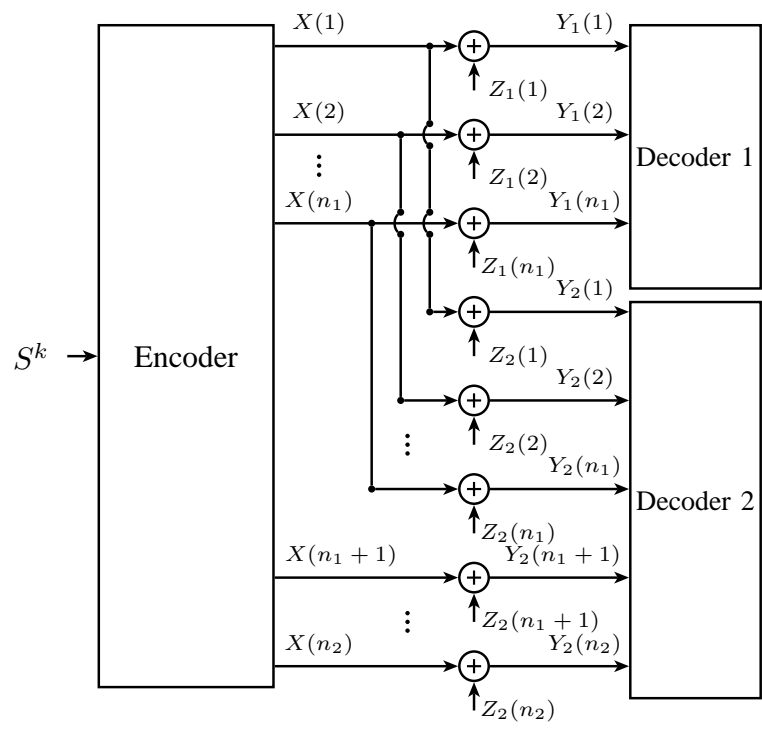

Fig. 1. Broadcasting a Gaussian source over a Gaussian broadcast channel with individual bandwidth mismatches. Here, the total number of channel uses, $n=\max \left(n_{1}, n_{2}\right)=n_{2}$.

sequence $X^{n}=(X(1), X(2), \ldots, X(n))$ where $X(l)$ denotes the $l^{\text {th }}$ channel input and $X^{n}$ satisfies a power constraint. Note here that our notation defines $X^{n}$ as the first $n$ channel symbols sent.

Let $Y_{i}(l)$ be the channel output observed by user $i$ on the $l^{\text {th }}$ channel use for $i \in\{1,2\}$ and $l=1,2, \ldots, n$. The channel model is given by

$$
Y_{i}(l)=X(l)+Z_{i}(l),
$$

where $Z_{i}(l)$ is the zero-mean additive Gaussian noise observed at user $i$ 's $l^{\text {th }}$ channel output. The channel is memoryless in the sense that $Z_{i}(l)$ is drawn i.i.d. from a $\mathcal{N}\left(0, N_{i}\right)$ distribution.

Now although the length $n$ channel input is broadcasted to both users, we will assume that due to each user's delay constraint, the $i^{\text {th }}$ user reconstructs the source after observing only the first $n_{i}$ channel input symbols, denoted as $X^{n_{i}}$, where $n_{i} \leq n$. Specifically, we have that $n=\max \left(n_{1}, n_{2}\right)$. The reconstruction's fidelity is measured with the squared error distortion

$$
d\left(s^{k}, \hat{s}^{k}\right)=\frac{1}{k} \sum_{j=1}^{k}(s(j)-\hat{s}(j))^{2} .
$$

Definition 1. A $\left(k, P, n_{1}, n_{2}, D_{1}, D_{2}\right)$ source-channel code for source $S$ on the Gaussian broadcast channel consists of

1) An encoding function $f: \mathbb{R}^{k} \rightarrow \mathbb{R}^{n}$ satisfying (1) $n=\max \left(n_{1}, n_{2}\right)$, (2) $X^{n}=f\left(S^{k}\right)$ and (3) $\frac{1}{n} \sum_{j=1}^{n} \mathbb{E}(X(j))^{2} \leq P$.
2) Two decoding functions $g_{i}: \mathbb{R}^{n_{i}} \rightarrow \mathbb{R}^{k}$ for $i \in$ $\{1,2\}$ such that $\mathbb{E} d\left(S^{k}, g_{i}\left(X^{n_{i}}+Z_{i}^{n_{i}}\right)\right) \leq D_{i}$ holds for $i \in\{1,2\}$.

where $\mathbb{E}(\cdot)$ is the expectation operation.

Now as mentioned in Section I, we associate with the $i^{\text {th }}$ user, a delay parameter $b_{i} \in[0, \infty)$, called the bandwidth expansion factor. This represents the number of channel uses per source symbol that are delivered over the $i^{\text {th }}$ user's channel. That is, the $i^{\text {th }}$ user requires that at most $b_{i} \cdot k$ channel uses be employed on his channel before he reconstructs $S^{k}$ subject to his distortion constraint. Our problem is now defined as characterizing the achievable distortion region under a given pair of bandwidth expansion factors as per the next definition.

Definition 2. A distortion pair $\left(D_{1}, D_{2}\right)$ is said to be $\left(b_{1}, b_{2}\right)$ achievable over the Gaussian broadcast channel under power constraint $P$, if for every $\delta>0$ there exists for sufficiently large $k, a\left(k, P, b_{1} \cdot k, b_{2} \cdot k, d_{1}, d_{2}\right)$ sourcechannel code such that

$$
D_{i}+\delta \geq d_{i}, \quad i \in\{1,2\} .
$$

The achievable distortion region is the set of all achievable distortion pairs under the prescribed bandwidth expansion pair.

\section{AN INNER BOUND FOR THE ACHIEVABLE DisTORTION REGION}

In this section, we propose several coding schemes that collectively provide an inner bound for the achievable distortion region. Assume that $N_{1}<N_{2}$. Since the performance of the Gaussian broadcast channel is identical to that of a degraded Gaussian broadcast channel, we will refer to user 1 as the strong user, and user 2 as the weak user. We specialize our analysis and focus on the case in which both users have bandwidth expanded and the strong user has the more stringent delay constraint, i.e., $1<b_{1}<b_{2}$. In this case, we will find particular values $b_{1}^{*}$ and $b_{2}$ in Section III-A, for which both users can simultaneously achieve distortions that are point-to-point optimal. In particular, we say that user $i$ achieves his point-to-point distortion at bandwidth expansion factor $b_{i}$, if he achieves a distortion $D_{i}^{*}$ given by

$$
D_{i}^{*}\left(b_{i}\right)=\frac{\sigma^{2}}{\left(1+\frac{P}{N_{i}}\right)^{b_{i}}}, \quad i \in\{1,2\},
$$

where (4) is obtained from substituting for the rate distortion and channel capacity functions of the Gaussian source/channel into the separation theorem. Since we will be interested in studying how the distortions of the 


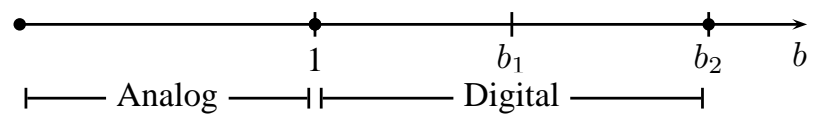

Fig. 2. The splitting into analog and digital branches in time.

two users depend on their bandwidth expansion factors, we use a slight abuse of notation and express $D_{i}^{*}$ as a function of only $b_{i}$. In the following sections, we will parameterize the problem by fixing any arbitrary $b_{2}$, while considering the distortions of both users as we vary $b_{1}$ relative to $b_{2}$.

\section{A. A Critical Bandwidth Expansion Value}

Consider first, the problem of coding entirely for the weak user so that he achieves his point-to-point distortion at some $b_{2}>1$. We will use a hybrid digital-analog coding scheme as in [1]-[3]. We first split our channel block of length $n=b_{2} k$ into a lower analog branch and an upper digital branch which are to be concatenated together, i.e., multiplexed in that order in time (see Fig. 2). The digital branch is of blocklength $k\left(b_{2}-1\right)$ and contains a coarse description of the source sequence $S^{k}$. Specifically, we perform vector quantization of the source at average distortion $D_{Q}$, which is set so that the quantizer rate is equal to the weak user's channel capacity on the digital branch, i.e.,

$$
\frac{1}{2} \log \left(\frac{\sigma^{2}}{D_{Q}}\right)=\frac{\left(b_{2}-1\right)}{2} \log \left(1+\frac{P}{N_{2}}\right) .
$$

Denote the output of the source quantizer as $S_{Q}^{k}$. We subtract $S_{Q}^{k}$ from the source sequence $S^{k}$, and the resultant quantization error $E$, is scaled and sent over the analog branch, which has blocklength $k$.

At the receiver, the weak user demultiplexes the analog and digital branches and first recovers the coarse description from the digital branch. He then obtains the linear minimum mean squared error (MMSE) estimate of the quantization error $\hat{E}$ from the analog branch, and reconstructs the source sequence as $\hat{S}^{k}=S_{Q}^{k}+\hat{E}$. It is readily verified that this scheme is point-to-point optimal for the weak user at $b_{2}$.

Now consider the strong user as all this transpires. He also demultiplexes the analog and digital branches. That is, he observes the first $k$ channel symbols and sets this aside as the analog branch and then observes the next $k\left(b_{2}-1\right)$ channel symbols as a digital branch. We note however, that the strong user can actually listen to only $k\left(b_{1}^{*}-1\right)<k\left(b_{2}-1\right)$ channel symbols after the analog branch and yet still recover the coarse description.

In the digital branch, note that the encoder employs a Gaussian codebook composed of $2^{k\left(b_{2}-1\right) C_{2}}$ length $k\left(b_{2}-1\right)$ codewords whose components are sampled i.i.d. from a $\mathcal{N}(0, P)$ distribution, where $C_{2}$ is the channel capacity for the weak user, i.e., $C_{2}=\frac{1}{2} \log (1+$ $\left.P / N_{2}\right)$. From the channel coding theorem, it is not hard to see that with arbitrarily small probability of error, the strong user has to listen to only $k\left(b_{1}^{*}-1\right)$ channel uses before being able to uniquely identify which codeword was sent by joint-typicality decoding, where $b_{1}^{*}$ satisfies $k\left(b_{1}^{*}-1\right) C_{1}=k\left(b_{2}-1\right) C_{2}$, i.e.,

$$
\frac{\left(b_{1}^{*}-1\right)}{2} \log \left(1+\frac{P}{N_{1}}\right)=\frac{\left(b_{2}-1\right)}{2} \log \left(1+\frac{P}{N_{2}}\right) \text {. }
$$

After recovering the channel codeword and thus the coarse description, the strong user can perform their own MMSE estimate of the quantization error to achieve a distortion of

$$
D_{1}=\frac{D_{Q}}{\left(1+\frac{P}{N_{1}}\right)} \text {. }
$$

Combining (4), (5), (6) and (7), we have that at $b_{1}^{*}$, the strong user can in fact achieve distortion $D_{1}=D_{1}^{*}\left(b_{1}^{*}\right)$.

Theorem 1. Let $b_{2}>1$. Then for some $b_{1}^{*}$ satisfying (6), the distortion pair $\left(D_{1}, D_{2}\right)=\left(D_{1}^{*}\left(b_{1}^{*}\right), D_{2}^{*}\left(b_{2}\right)\right)$ is $\left(b_{1}^{*}, b_{2}\right)$ achievable.

Again, it is worth mentioning that the result of both users achieving their point-to-point distortion in Theorem 1 is not possible in the familiar problem where $b_{1}=b_{2}$. Naturally, the question now arises as to what the achievable distortions would be if we deviate from these critical bandwidth expansion factors. We explore this in the next two sections.

\section{B. A Successive Refinement Coding Scheme}

In this section, we consider the case that the delay of the strong user, $b_{1}$, is even stricter than that given by $b_{1}^{*}$, i.e., $1<b_{1}<b_{1}^{*}<b_{2}$. In this case, we will use a "successive refinement" strategy consisting of the coding scheme of Section III-A followed by Wyner-Ziv coding to show that both users can still simultaneously achieve point-to-point optimal distortions.

Now given that $b_{1}<b_{1}^{*}$, we first find a $\tilde{b}_{2}$ satisfying (6) when $b_{1}^{*}$ and $b_{2}$ are replaced by $b_{1}$ and $\tilde{b}_{2}$ respectively. In other words, we find a $\tilde{b}_{2}$ such that $k\left(b_{1}-1\right) C_{1}=$ $k\left(\tilde{b}_{2}-1\right) C_{2}$, i.e.,

$$
\frac{\left(b_{1}-1\right)}{2} \log \left(1+\frac{P}{N_{1}}\right)=\frac{\left(\tilde{b}_{2}-1\right)}{2} \log \left(1+\frac{P}{N_{2}}\right) \text {. }
$$

Operationally, this is equivalent to employing the coding scheme of Section III-A with a coarse description that achieves distortion $\tilde{D}_{Q}$ using rate $(1 / 2) \log \left(\sigma^{2} / \tilde{D}_{Q}\right)=\left(\tilde{b}_{2}-1\right) \log \left(1+P / N_{2}\right)$. Here $\tilde{b}_{2}$ is chosen as in (8) and from Theorem 1, we achieve 
the point-to-point optimal distortions for both user 1 and user 2 at $b_{1}$ and $\tilde{b}_{2}$ respectively. To show that we can also be optimal for user 2 at $b_{2}$, we use Wyner-Ziv coding immediately after the coding scheme of Section III-A. Specifically, after $k \tilde{b}_{2}$ channel uses, we send a WynerZiv bit stream for the weak user assuming that he has side information at a mean squared error of $D_{2}^{*}\left(\tilde{b}_{2}\right)$ with respect to the source. Note here that we can invoke the upper bound on the quadratic Wyner-Ziv rate-distortion function as in [2], to show that in order to achieve a new distortion of $D_{2}$, a bit stream of rate $R^{\mathrm{WZ}}\left(D_{2}\right)$ is sufficient, where

$$
R^{\mathrm{WZ}}\left(D_{2}\right)=\frac{1}{2} \log \frac{D_{2}^{*}\left(\tilde{b}_{2}\right)}{D_{2}} .
$$

Setting (9) equal to the channel capacity of the weak user's remaining digital branch, we get that

$$
\frac{1}{2} \log \frac{D_{2}^{*}\left(\tilde{b}_{2}\right)}{D_{2}}=\frac{\left(b_{2}-\tilde{b}_{2}\right)}{2} \log \left(1+\frac{P}{N_{2}}\right) .
$$

Combining (4), (8) and (10) and rearranging for $D_{2}$, we have that after sending the Wyner-Ziv bit stream, we can in fact achieve $D_{2}=D_{2}^{*}\left(b_{2}\right)$ at $b_{2}$.

Theorem 2. Let $b_{2}>1$ and let $b_{1}^{*}$ satisfy (6) for the choice of $b_{2}$. Then for any $b_{1}$ such that $1<b_{1} \leq b_{1}^{*}$, the distortion pair $\left(D_{1}, D_{2}\right)=\left(D_{1}^{*}\left(b_{1}\right), D_{2}^{*}\left(b_{2}\right)\right)$ is $\left(b_{1}, b_{2}\right)$ achievable.

As an interesting aside, from (6) we have that in the limit of high SNR $\left(P \gg N_{1}\right), b_{1}^{*}=b_{2}-o_{P}(1)$ where $o_{P}(1)$ goes to zero as $P \rightarrow \infty$. Theorem 2 then states that we can nearly achieve point-to-point optimality for both users for any $b_{1}<b_{2}$ under bandwidth expansion.

\section{An Achievable Tradeoff}

In this section, we present an achievable tradeoff in distortion pairs for when $1<b_{1}^{*}<b_{1}<b_{2}$. We will again use a hybrid-digital analog scheme, however this time, we will further divide the digital branch (in time) into two regions. Region I will include the channel uses from $k$ to $k b_{1}$ while Region II will include the channel uses from $k b_{1}$ to $k b_{2}$ (see Fig. 3). Following this division, we now view the digital branch analogously to the product of two unmatched degraded broadcast channels [6]. Here, Region I corresponds to a degraded brodcast channel in which user 1 is the stronger user, while Region II corresponds to another broadcast channel in which user 2 is the stronger user and user 1 has infinite noise power. We will use these two broadcast channels to send a common message intended for both users, and a private message intended only for user 1; cf. [2], [3].

We denote the common and private message rates, measured in bits per source symbol, as $R_{0}$ and $R_{1}$

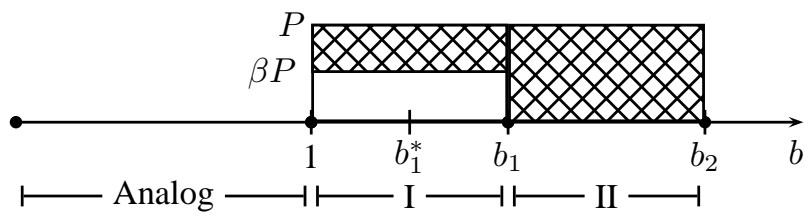

Fig. 3. The splitting into analog and digital branches. The digital branch is further split into Region I and Region II and the power in it (shown vertically) is allocated between a common message (crosshatched) and a private message.

respectively. As the private message is intended only for user 1 , we can send it only over Region I. We will dedicate a portion $\beta \in[0,1]$ of the total power in Region I for this purpose. Specifically, we set

$$
R_{1}=\frac{b_{1}-1}{2} \log \left(1+\frac{\beta P}{N_{1}}\right) .
$$

The common message is a coarse description of the source. It is sent over both regions, however only a portion $(1-\beta)$ of the power in Region I will be used for this purpose while the full power of Region II will be used. Specifically, we perform vector quantization of the source at an average distortion $\hat{D}_{Q}$, set so that

$$
\begin{aligned}
R_{0}=\frac{1}{2} \log \left(\frac{\sigma^{2}}{\hat{D}_{Q}}\right) & =\frac{b_{1}-1}{2} \log \left(1+\frac{(1-\beta) P}{N_{2}+\beta P}\right) \\
& +\frac{b_{2}-b_{1}}{2} \log \left(1+\frac{P}{N_{2}}\right) .
\end{aligned}
$$

Equation (12) makes it clear that user 2 will be able to recover the coarse description by treating the private message as noise in Region I, and then recovering the rest of the message in Region II. In order for user 1 to also recover the message in Region I, we require that

$$
R_{0} \leq \frac{b_{1}-1}{2} \log \left(1+\frac{(1-\beta) P}{N_{1}+\beta P}\right) .
$$

After each user recovers the coarse description, they again estimate the quantization error over the analog branch, after which, user $i$ can achieve distortion $\tilde{D}_{i}$ of

$$
\tilde{D}_{i}=\frac{\hat{D}_{Q}}{1+\frac{P}{N_{i}}}, \quad i \in\{1,2\} .
$$

Since this is all we will be sending to the weak user, $\tilde{D}_{2}$ is the final distortion user 2 achieves. We will however, send extra Wyner-Ziv bits for user 1 in Region I assuming that he already has side information at distortion $\tilde{D}_{1}$ relative to the source. The extra bits help user 1 achieve a distortion $D_{1}$, where we set $D_{1}$ so that the Wyner-Ziv rate equals the private message rate, i.e.,

$$
R^{\mathrm{WZ}}\left(D_{1}\right)=\frac{1}{2} \log \frac{\tilde{D}_{1}}{D_{1}}=R_{1} .
$$

Suppose for now that user 1 is to be optimal at $b_{1}$. In this case, we see that (13) must be met with equality. To 


$$
\begin{aligned}
& \mathcal{D}_{1}(\beta) \triangleq \frac{\sigma^{2}}{\left(1+\frac{(1-\beta) P}{N_{2}+\beta P}\right)^{b_{1}-1}\left(1+\frac{P}{N_{2}}\right)^{b_{2}-b_{1}}\left(1+\frac{P}{N_{1}}\right)\left(1+\frac{\beta P}{N_{1}}\right)^{b_{1}-1}} \\
& \mathcal{D}_{2}(\beta) \triangleq \frac{\sigma^{2}}{\left(1+\frac{(1-\beta) P}{N_{2}+\beta P}\right)^{b_{1}-1}\left(1+\frac{P}{N_{2}}\right)^{b_{2}-b_{1}+1}} .
\end{aligned}
$$

this end, define $\beta_{0}$ as the value of $\beta$ that accomplishes this. Combining (12) and (13), we have that

$$
\beta_{0}=\frac{\left(1+\frac{P}{N_{1}}\right)-\left(1+\frac{P}{N_{2}}\right)^{\frac{b_{2}-1}{b_{1}-1}}}{\frac{P}{N_{1}}\left(1+\frac{P}{N_{2}}\right)^{\frac{b_{2}-1}{b_{1}-1}}-\left(1+\frac{P}{N_{1}}\right) \frac{P}{N_{2}}} .
$$

It can be verified that $0<\beta_{0}<1$ whenever $1<b_{1}^{*}<$ $b_{1}<b_{2}$, which is the region of interest and so $\beta_{0}$ is indeed a valid power allocation. Defining the functions $\mathcal{D}_{1}(\beta)$ and $\mathcal{D}_{2}(\beta)$ as in (16) and (17), after combining (11), (12), (14) and (15) for user 1 and (12) and (14) for user 2 , we have that the distortion pair $\left(\mathcal{D}_{1}\left(\beta_{0}\right), \mathcal{D}_{2}\left(\beta_{0}\right)\right)$ is achievable. In particular, since the right hand sides of (12) and (13) are equal when $\beta=\beta_{0}$, we can confirm that user 1 is optimal at $b_{1}$ for this power allocation.

Let us now consider the scenario if we did not require user 1 to be optimal at $b_{1}$. To this end, we restrict $\beta$ by setting $\beta=\alpha \beta_{0}$ for some $\alpha \in[0,1]$. In particular, if $\alpha=1$, we recover $\beta=\beta_{0}$ as our power allocation, and user 1 is optimal. As we decrease $\alpha$ (and in turn $\beta$ ), we introduce more slackness in (13) until $\beta=0$. This makes the coding identical to that in Section III-A, so that the weak user is optimal. In general, we may achieve a tradeoff in distortions by letting $\alpha$ vary in $[0,1]$.

Theorem 3. Let $b_{2}>1$ and let $b_{1}^{*}$ satisfy (6) for the choice of $b_{2}$. Then for any $\alpha \in[0,1]$ and for any $b_{1}$ such that $1<b_{1}^{*}<b_{1}<b_{2}$, the distortion pair $\left(D_{1}, D_{2}\right)=$ $\left(\mathcal{D}_{1}\left(\alpha \beta_{0}\right), \mathcal{D}_{2}\left(\alpha \beta_{0}\right)\right)$ is $\left(b_{1}, b_{2}\right)$ achievable, where $\beta_{0}$ is given by (18)

We remark that as $b_{1} \rightarrow b_{2}$, from (18) we have that $\beta_{0} \rightarrow 1$. In this case, the coding scheme of this section reduces to the one proposed in [2]. In light of the results in [3] and [6] we also suggest that the results in this section may be improved by further allocating power amongst the digital and analog branches and subbranches, introducing a private message to user 2 and optimizing over the boundaries of Regions I and II however for conciseness, we do not pursue this here. Finally, we mention that the outer bound in [2] using $b=\max \left(b_{1}, b_{2}\right)=b_{2}$ may also be applied here since this bound would be obeyed a fortiori when $b_{1}<b_{2}$.

Theorem 3 completes our coding scheme for any $b_{1}$ and $b_{2}$ such that $1<b_{1}<b_{2}$. Fig. 4 plots our achievable

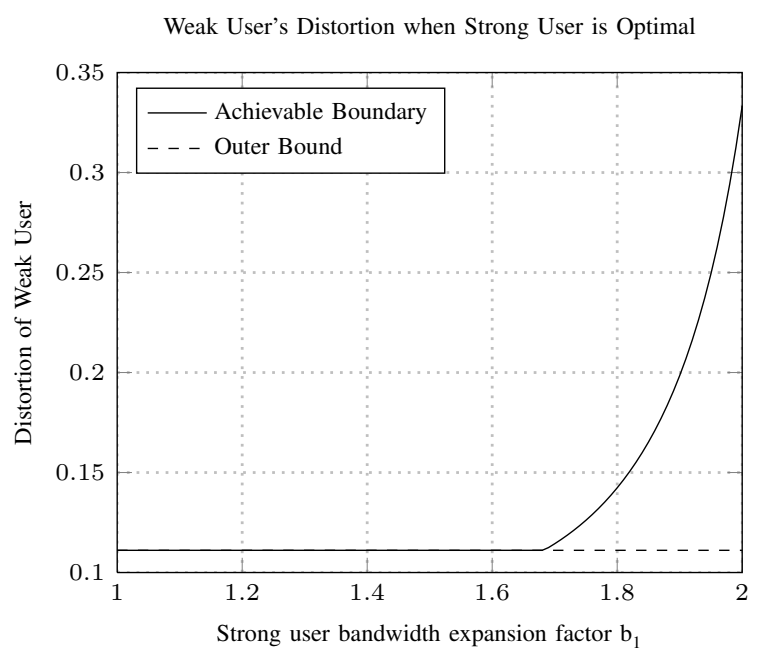

Fig. 4. Distortion of the weak user when the strong user is optimal at $b_{1}$. We vary $b_{1}$ and fix $b_{2}=2, \mathrm{SNR}_{1}=6.02 \mathrm{~dB}, \mathrm{SNR}_{2}=3.01 \mathrm{~dB}$, $\sigma^{2}=1$.

scheme for the weak user when the strong user is always optimal at $b_{1}$ and we vary $b_{1}$ relative to a fixed $b_{2}$. As is readily seen, the weak user is also able to achieve an optimal distortion, shown as the dashed line, until a critical bandwidth expansion factor $b_{1}^{*}$. After this critical value, the requirement that the strong user is optimal results in an increasing distortion for the weak user.

\section{REFERENCES}

[1] U. Mittal and N. Phamdo, "Hybrid digital-analog (hda) joint source-channel codes for broadcasting and robust communications," IEEE Trans. Inf. Theory, vol. 48, no. 5, pp. 1082-1102, May 2002.

[2] Z. Reznic, M. Feder, and R. Zamir, "Distortion bounds for broadcasting with bandwidth expansion," IEEE Trans. Inf. Theory, vol. 52, no. 8, pp. 3778-3788, Aug. 2006.

[3] V. M. Prabhakaran, R. Puri, and K. Ramchandran, "Hybrid digitalanalog codes for source-channel broadcast of gaussian sources over gaussian channels," IEEE Trans. Inf. Theory, vol. 57, no. 7, pp. 4573-4588, Jul. 2011.

[4] Y. Li and E. Soljanin, "Rateless codes for single-server streaming to diverse users," in Proc. 47th Annual Allerton Conference on Communication, Control, and Computing, Montecello, IL, Jan. 2009, pp. 1419-1426.

[5] L. Tan, MASc. thesis, Dept. Elect. Eng., Univ. Toronto, Toronto, ON, In preparation 2012.

[6] A. E. Gamal, "The capacity of the product and sum of two unmatched broadcast channels," Problemy Perdachi Informatsi, vol. 16, no. 1, pp. 3-23, Jan.-Mar. 1980. 